\title{
Cerebroprotein hydrolysate- new paradigm in management of neurological problems
}

Volume 2 Issue 6 - 2015

\section{Editorial}

Neuroscientists have taken great efforts to develop drugs to stimulate areas of the brain to repair itself by replacing its own cells. ${ }^{1}$ Neurotrophic factors are small proteins that exert survivalpromoting and trophic actions on neuronal cells. These neurotrophic factors are NGF (nerve growth factor), GDNF (glial cell-derived neurotrophic factor), BDNF (brain-derived neurotrophic factor), NT-3 (neurotrophin-3), GAP-43 (growth associated protein 43) and CNFT (cilliary neurotrophic factor). ${ }^{2}$ Drugs like edaravone, citicoline, and piracetam have been developed based on these neurotrophic factors.

Glial cells continue to undergo cell division in adulthood and their significant proliferative ability is seen after brain injury. ${ }^{3}$ Though neurons don't divide, they undergo a lot of activity after injury. Treatment of numerous neurodegenerative disorders is changing at an astonishing pace. It may be noted that neurons attempt to re-wire the brain following an injury, as the neurons in the adult brain have remarkable capacity for remodelling away from the actual injury. ${ }^{4}$

Cerebroprotein hydrolysate is an unique neurotrophic peptidergic mixture produced by standardized enzymatic breakdown of lipid-free porcine brain proteins.. It acts like endogenous neurotrophic factors as it consists of short biological peptides. Neurotrophic activity can be detected within 1 day after a single injection. ${ }^{5}$

Cerebroprotein Hydrosylate enhances neurogenesis, neuronal survival, provides neuromodulatory action, increases/modulates neuronal plasticity and neuronal repair and has neuroimmunotrophic actions and thus has a unique neurotrophic activity. ${ }^{6}$ Cerebroprotein hydrosylate helps in Neuronal differentiation and protection against ischaemic and neurotoxic lesions. It regulates and improves neuronal metabolism. It reduces excitotoxic damage, blocks over-activation of calcium dependent proteases, and scavenges free oxygen radicals. It has been found in animal studies that early intervention with cerebroprotein hydrosylate reduces blood-brain and bloodcerebrospinal fluid barrier permeability changes, attenuates brain pathology and brain edema, and mitigates functional deficits caused by traumatic brain injury. ${ }^{7}$ It improved brain bioelectrical activity, i.e. reduced EEG ratio by increasing fast frequencies and reducing slow activities and also improves cognitive performance in tasks, evaluating attention and memory functions in post acute traumatic brain injury patients. ${ }^{8}$

Neuronal survival enhancement is produced through effect on calpain. Calpain hyper-activation is implicated in a number of neurodegenerative disorders. Cerebroprotein hydrolysate inhibits Calpian. Neuro-immunotrophic activity is produced by inhibition of microglial activation and expression of IL-1 beta. This reduces inflammation. Neuromodulatory effect is produced by increasing GLUT- 1 expression. GLUT-1 is responsible for more than $90 \%$ of glucose transport to brain. ${ }^{9}$ Neuronal plasticity is produced by reduction of amyloid beta accumulation, increased MAP 2 and synaptophysin synthesis.

\author{
Arun Oommen \\ Consultant Neurosurgeon, Lakeshore Hospital and Research \\ Centre, India
}

\begin{abstract}
Correspondence: Dr. Arun Oommen, MBBS, MS (Gen Surg), Mch (Neurosurgery), MRCS Ed, MBA, Consultant Neurosurgeon, Lakeshore Hospital and Research Centre, Kochi,
\end{abstract} India,Email oommenarun@yahoo.com

Received:September 26, 2015 | Published: October 0I, 2015

Cerebroprotein hydrolysate has been found to be useful in Traumatic brain injury, acute ischaemic stroke, Vascular dementia, Extrapontine myelinolysis and Alzheimer's disease (AD). ${ }^{10-12}$

There are very few medications that can reduce the functional disability caused by traumatic brain injury. The complex study of cognitive and emotional status, levels of serum serotonin and brainderived neurotrophic factor (BDNF) performed in 72 patients with acute traumatic brain injury, with a special focus on moderate brain injuries (MBI), treated with Cerebrolysin found that cerebrolysin improves outcomes of closed craniocerebral injury by promoting activation of neurotrophic processes. ${ }^{13}$ Cerebroprotein hydrolysateaugmented proliferation, differentiation, migration of adult SVZ neural progenitor cells results in increased number of neural progenitor cells and neuroblasts which contribute to neurogenesis. The beneficial effect seen in traumatic brain injury and acute ischaemic stroke may be due to this mechanism. A double-blind, placebo-controlled, randomized study showed that Cerebrolysin improves the cognitive function of patients with mild traumatic brain injury (MTBI) at 3rd month after injury, especially for long-term memory and drawing function tested on Mini-Mental Status Examination (MMSE) and Cognitive Abilities Screening Instrument (CASI) scores. ${ }^{14}$

Cerebroprotein hydrolysate demonstrates noticeable improvements in clinical global impression, cognitive performance and on level of activities of daily living in patients suffering from Alzheimer's and vascular dementia. ${ }^{15,16}$ Mechanism for its beneficial effect in Alzheimer's disease can be because it counteracts the negative effect of the elevated FGF-2 on neurogenesis and neuromodulation. ${ }^{17}$ For treatment of dementia we have no other drugs available which act at neuronal level. ${ }^{18}$ For treatment of dementia only few medical options are available like acetylcholinesterase inhibitors (donepezil) and $\mathrm{N}$-methyl-D-aspartate (NMDA) receptor blockers (memantine). 
Cerebroprotein hydrolysate has not been reported to produce serious side effects. Few side effects like nausea, headache, vertigo, prespiration, confusion, irritability, fever, hallucinations, etc. has been reported. It is contraindicated in hypersensitivity, epilepsy and severe renal impairment. It should be used with caution in pregnant and lactating ladies as the safely profile is still not established. ${ }^{19,20}$

Cerebroprotein hydrolysate is a medication that acts at a brain level and provides us with an effective tool for improving levels of activities of daily living in such patients with various neurological disorders and decreasing their dependence on caregivers though further research in larger populations and clinical trials is warranted. Initial experiences shows promising results for Cerebro protein hydrosylate but it is still in its early stages and will require extensive randomized controlled trials before its efficacy is proved.

\section{Acknowledgments}

None.

\section{Conflicts of interest}

None.

\section{References}

1. Thomas SV. Addressing problems of dementia in India. Annals of Indian Academy of Neurology. 2011;14(3):147.

2. http://www.ceregene.com/neurotrophic.asp

3. Barrett KE, Boitano S, Barman SM, et al. Ganong's Review of Medical Physiology. (23rd edn), McGraw Hill Lange, USA. 2010.

4. Gopalan T. Neurons Key To Healing Of Brain Injuries Identified. Research News, India, August 24, 2010.

5. Hartbauer M, Hutter-Paier B, Skofitsch G, et al. Antiapoptotic effects of the peptidergic drug cerebrolysin on primary cultures of embryonic chick cortical neurons. J Neural Transm. 2001;108(4):59-73.

6. Sharma HS, Zimmermann-Meinzingen S, Johanson CE. Cerebrolysin reduces blood-cerebrospinal fluid barrier permeability change, brain pathology, and functional deficits following traumatic brain injury in the rat. Ann N Y Acad Sci. 2010;1199:125-137.

7. Wong GK, Zhu XL, Poon WS. Beneficial effect of cerebrolysin on moderate and severe head injury patients: result of a cohort study. Acta Neurochir Suppl. 2005;95:59-60.
8. Alvarez XA, Sampedro C, Pérez P, et al. Positive effects of cerebrolysin on electroencephalogram slowing, cognition and clinical outcome in patients with postacute traumatic brain injury: an exploratory study. Int Clin Psychopharmacol. 2003;18(5):271-278.

9. Boado RJ, Wu D, Windisch M. In vivo upregulation of the blood-brain barrier GLUT1 glucose transporter by brain-derived peptides. Neurosci Res. 1999;34(4):217-224.

10. Karia S, Mahajan PT, Shah N, et al. Cerebroprotein hydrolysate in traumatic brain injury. El Mednifico Journal. 2014;2:34-35.

11. Karia S, Majlikar R, De Sousa A, et al. Cerebroprotein hydrolysate in Extra-Pontine Myelinosis - A Case Report. International Journal of Scientific and Research Publication. 2014;4(1):1-2.

12. Gupta P, Yadav S, Singal KK. Cerebroprotein hydrolysate: Innovation in the treatment of neurodegenerative disorders. Journal of Indian Academy of Clinical Medicine. 2014;15(2):132-133.

13. Selianina NV, Karakulova IV. The effect of neurotrophic treatment on the activation of reparative processes in patients with acute traumatic brain injury. Zh Nevrol Psikhiatr Im S S Korsakova. 2012;112(5):46-49. [Article in Russian].

14. Chen CC, Wei ST, Tsaia SC, et al. Cerebrolysin enhances cognitive recovery of mild traumatic brain injury patients: double-blind, placebocontrolled, randomized study. Br J Neurosurg. 2013;27(6):803-807.

15. http://www.superhumanstore.com/shg/cerebrolysin- $\% \mathrm{E} 2 \% 80 \% 93-\mathrm{a}-$ unique-treatment-option-for-alzheimer $\% \mathrm{E} 2 \% 80 \% 99 \mathrm{~s}$-disease/

16. Allegri RF, Guekht A. Cerebrolysin improves symptoms and delays progression in patients with Alzheimer's disease and vascular dementia. Drugs Today (Barc). 2012;48(Suppl A):25-41.

17. Chen H, Tung YC, Li B, et al. Trophic factors counteract elevated FGF-2-induced inhibition of adult neurogenesis. Neurobiol Aging. 2007;28(8):1148-1162.

18. Alvarez XA, Cacabelos R, Sampedro C, et al. Efficacy and safety of Cerebrolysin in moderate to moderately severe Alzheimer's disease: results of a randomized, double-blind, controlled trial investigating three dosages of Cerebrolysin. Eur J Neurol 2011. 2011;18(1):59-68.

19. http://en.cnki.com.cn/Article_en/CJFDTOTAL-XYYL606.002.htm

20. Man SC, Chan KW, Lu JH, et al. Systematic Review on the Efficacy and Safety of Herbal Medicines for Vascular Dementia. Evid Based Complement Alternat Med. 2012;2012:426215. 